\title{
A Randomized Trial Evaluating the Effect of 10 versus 20 Revolutions Inside the Lymph Node on the Diagnostic Yield of EBUS-TBNA in Subjects with Sarcoidosis
}

\author{
Sahajal Dhooria ${ }^{a}$ Inderpaul Singh Sehgal ${ }^{a}$ Nalini Gupta ${ }^{b}$ Amanjit Bal $^{c}$ \\ Kuruswamy Thurai Prasad ${ }^{a}$ Ashutosh Nath Aggarwal ${ }^{a}$ Babu Ram $^{\mathrm{a}}$ \\ Ritesh Agarwal ${ }^{a}$ \\ aDepartment of Pulmonary Medicine, Postgraduate Institute of Medical Education and Research, Chandigarh, India; \\ ${ }^{b}$ Department of Cytology and Gynecologic Pathology, Postgraduate Institute of Medical Education and Research, \\ Chandigarh, India; ' Department of Histopathology, Postgraduate Institute of Medical Education and Research, \\ Chandigarh, India
}

\section{Keywords}

Endosonography · EBUS-TBNA · Tuberculosis · Granuloma

\begin{abstract}
Background: Endobronchial ultrasound (EBUS)-guided transbronchial needle aspiration (TBNA) is the preferred modality for sampling intrathoracic lymph nodes in patients with suspected sarcoidosis. Whether the number of revolutions of the needle inside the lymph node while performing TBNA affects the diagnostic yield is unknown. Objectives: The aim of this paper was to compare the yield of different numbers of needle revolutions (10 vs. 20) during EBUS-TBNA in sarcoidosis. Methods: Consecutive subjects with a clinicoradiological suspicion of sarcoidosis were randomized 1:1 to undergo EBUS-TBNA with either 10 (group 1) or 20 revolutions (group 2). The primary and secondary outcomes were the diagnostic yield and adequacy of aspirates, respectively. Other outcomes were procedure duration, gross blood contamination of the aspirates, and safety of the procedure. $\boldsymbol{R} \boldsymbol{e}$ sults: Of the 171 subjects screened, 150 (mean age 43.5 years; $47.3 \%$ women) were randomized. A mean of 2.8 (group
\end{abstract}

$1: 2.8$, group $2: 2.7 ; p=0.37$ ) lymph nodes were sampled per subject with a mean of 2.1 passes per node in each group $(p=0.60)$. Among 133 subjects finally diagnosed with sarcoidosis, there was no difference $(p=0.65)$ in the diagnostic yield of EBUS-TBNA between group 1 (52/65, 80.0\%) and group 2 (57/68, 83.8\%). Adequate aspirates were obtained in 96.9 and $97.1 \%$ of the subjects in groups 1 and 2, respectively $(p=1.00)$. There was no difference in the procedure duration, the proportion of subjects with grossly bloody specimens, or complications between the 2 groups. Conclusions: The diagnostic yield and specimen adequacy were not different when EBUS-TBNA was performed with 10 or 20 revolutions in subjects with sarcoidosis.

(C) 2018 S. Karger AG, Basel

\section{Introduction}

Sarcoidosis is a systemic granulomatous disorder of an unknown cause [1]. As it most often involves the lungs and lymph nodes, these 2 sites are frequently accessed for a histologic diagnosis of the disease. Presently, endobron-

\section{KARGER}

(c) 2018 S. Karger AG, Basel 
chial ultrasound (EBUS)-guided transbronchial needle aspiration (TBNA) is extensively used for sampling intrathoracic lymph nodes in patients suspected to have sarcoidosis. Because of its high yield and excellent safety, EBUS-TBNA has gradually supplanted other modalities such as conventional TBNA and mediastinoscopy for this purpose [2-5]. The pooled yield of EBUS-TBNA in sarcoidosis is 79\% (range, 54-93) [6]. When combined with endobronchial biopsy (EBB) and transbronchial lung biopsy (TBLB), the yield may be as high as 92\% [7-9].

Due to the wide variation in the diagnostic yield of EBUS-TBNA in subjects with sarcoidosis $[6,10]$, an important question arises, whether certain technical aspects of the procedure affect the yield. In this context, several factors such as the number of lymph nodes sampled, number of passes made, use of a larger bore (19G and $21 \mathrm{G}$ vs. $22 \mathrm{G}$ ) aspiration needle, and the utilization of rapid onsite evaluation (ROSE) have been investigated $[8,9$, 11-13]. However, more research is required to delineate the utility of several other technical aspects of the procedure, and a call has been made for the same by 2 international societies $[14,15]$. While performing EBUS-TBNA, the needle is used to puncture a lymph node under realtime ultrasonographic guidance. Subsequently, back and forth movements of the needle (referred to as revolutions, agitations, excursions, or, sometimes, jabs) are performed to induce the transfer of the lymph node material into the lumen of the needle helped by either only capillary action or application of suction in addition to the capillary action [16]. These revolutions are likely to be instrumental in pushing the lymph node material into the needle lumen (assisted by suction and capillary action). Whether the number of revolutions made during the procedure affects the diagnostic yield remains unknown. We hypothesized that the use of 20 revolutions would produce a higher diagnostic yield of the procedure. In this study, we explore the effect of the number of revolutions (10 or 20) performed during intrathoracic lymph node sampling with EBUS-TBNA on the diagnostic yield in subjects suspected to have sarcoidosis.

\section{Methods}

This was a randomized controlled trial conducted between $\mathrm{Au}$ gust 2016 and September 2017 in the bronchoscopy suite of the Postgraduate Institute of Medical Education and Research, Chandigarh, India. The study protocol was approved by the Institute Ethics Committee (NK/2232/study/1116), and a written informed consent was obtained from all subjects. The trial was registered at www.clinicaltrials.gov (NCT02875756).

Needle Revolutions During EBUS-TBNA

\section{Patient Selection}

We included consecutive subjects with clinical and radiological suspicion of sarcoidosis if they met all the following criteria: (a) age between 18 and 85 years; (b) enlarged hilar and/or mediastinal lymph nodes $>10 \mathrm{~mm}$ (any axis) on computed tomography (CT) of the chest; and, (c) planned for EBUS-TBNA. Subjects were excluded if any of the following was present: (a) hypoxemia $\left(\mathrm{SpO}_{2}\right.$ $<92 \%$ on $\mathrm{FIO}_{2} \geq 0.3$ ); (b) treatment with systemic glucocorticoids for $>2$ weeks in the preceding 3 months; (c) diagnosis of sarcoidosis possible with another minimally invasive technique such as skin biopsy or peripheral lymph node sampling; or, (d) failure to provide informed consent.

\section{Randomization}

Subjects were randomized 1:1 to undergo EBUS-TBNA with the performance of either 10 (group 1) or 20 revolutions (group 2) inside the lymph node during every pass (Fig. 1). The computergenerated randomization sequence was placed in sealed opaque envelopes that were opened sequentially immediately before the procedure. The subject and the cytologist were blinded to the group assignment.

\section{Study Procedure}

Before EBUS-TBNA, the subjects underwent clinical examination and standard laboratory tests including a tuberculin skin test, spirometry, chest radiography, and CT of the chest.

All bronchoscopic procedures were performed in an outpatient setting under monitored conscious sedation by consultants experienced in the procedure or fellows supervised by the consultants. Premedication, topical anesthesia, and sedatives were administered using a standard protocol, as described previously [17, 18]. EBUS-TBNA was performed orally in the supine position using an EBUS bronchoscope (BF-UC180F; Olympus Medical, Japan) and a compatible ultrasound image processor (EU-ME1; Olympus Medical) [19]. Enlarged lymph nodes were identified, and the size of the lymph nodes was measured [20]. The lymph nodes were aspirated using the jabbing method under real-time ultrasound control with a dedicated 22G EBUS-TBNA aspiration needle (Vizishot, NA-201 SX-4022A; Olympus Medical). Continuous suction was applied with a $20-\mathrm{mL}$ Vacloc syringe while the catheter was moved back and forth for either 10 or 20 times according to the allocated study group. ROSE was not available. Two or more lymph node stations were accessed, and at least 2 passes were obtained from each lymph node sampled. Conventional bronchoscopy procedures, including EBB and TBLB, were performed at the discretion of the bronchoscopist $[7,21]$.

\section{Specimen Preparation}

Slides prepared from the EBUS-TBNA aspirates were either air-dried (for Ziehl-Neelsen staining) or immediately fixed in $95 \%$ alcohol (for cytological examination). The aspirate was also sent in saline for mycobacterial culture and Xpert MTB/RIF (Cepheid, Sunnyvale, CA, USA) [22].

\section{Diagnosis of Sarcoidosis}

Sarcoidosis was diagnosed on the basis of compatible clinical and radiological presentation, presence of nonnecrotic granulomas in any of the bronchoscopic specimens (EBUS-TBNA, EBB, or TBLB), and exclusion of similar presenting conditions [19, 23]. When granulomas were not demonstrated, a diagnosis of sarcoid- 


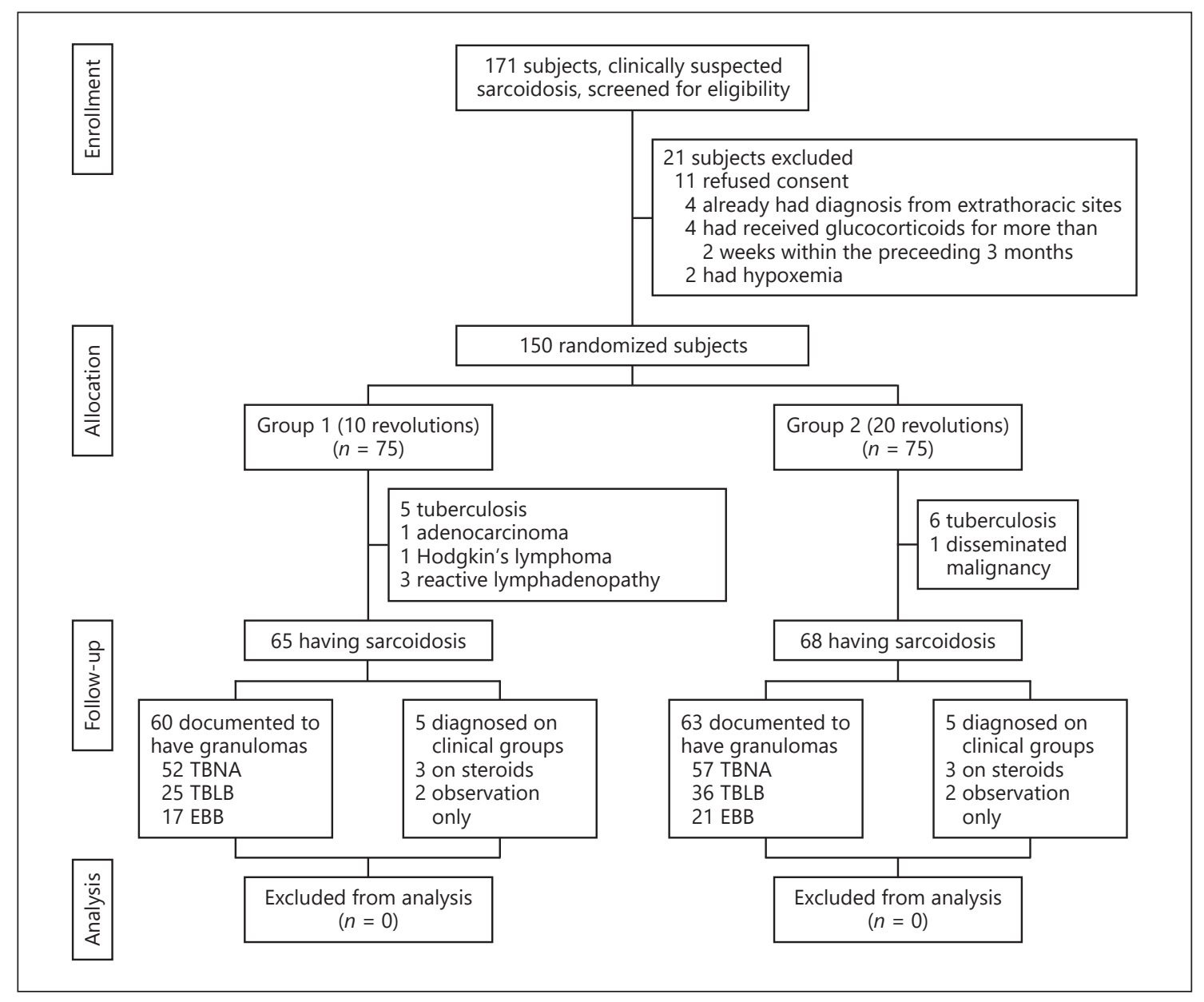

Fig. 1. CONSORT diagram showing the flow of the participants in the study.

osis was made based on the presence of a consistent clinicoradiological presentation, with no alternate diagnosis made at the 6-month follow-up, and there was either spontaneous regression of the symptoms (and/or lymph nodes) or good response to empiric glucocorticoid therapy [24].

\section{Endpoints}

The primary endpoint was the diagnostic yield (sensitivity) of EBUS-TBNA in subjects finally diagnosed to have sarcoidosis. The secondary endpoint was adequacy of the samples. A specimen was termed as diagnostic if it showed epithelioid cell granulomas and as adequate if the aspirate was either diagnostic or showed preponderance of lymphocytes.

\section{Sample Size}

The sample size was calculated based on a reported yield of $75 \%$ in group $1[7,8]$ and a presumed increase in yield by $10 \%$ in group 2 (i.e., 85\%). With these assumptions, 270 subjects would be required in each group (confidence level [1- $\alpha] 95 \%$, power level [1- $\beta$ ] $80 \%$ ) to detect this difference. As this sample size was not logistically feasible, a convenience sample of 150 subjects was chosen.

\section{Statistical Analysis}

Data are presented as means (standard deviation [SD]) or number (percentage). The commercial statistical package SPSS (version 22.0; IBM Inc., United States) was used for performing all data analysis. The $\chi^{2}$ test (or Fisher's exact test) or Student $t$ test was used to analyze differences between categorical and continuous variables, respectively. A $p$ value $<0.05$ was considered significant.

\section{Results}

Of the 171 screened subjects (Fig. 1), 150 were randomized to undergo EBUS-TBNA with either $10(n=75)$ or 20 revolutions $(n=75)$. The baseline characteristics were similar between the 2 groups (Table 1). There was no significant difference in the mean size of the lymph nodes sampled. The most common lymph node sampled 
Table 1. Baseline characteristics of the study population

\begin{tabular}{|c|c|c|c|c|}
\hline & $\begin{array}{l}\text { Group } 1 \\
(n=75)\end{array}$ & $\begin{array}{l}\text { Group } 2 \\
(n=75)\end{array}$ & $\begin{array}{l}\text { Total } \\
(n=150)\end{array}$ & $\begin{array}{l}p \\
\text { value }\end{array}$ \\
\hline Age, years & $45.3 \pm 11.9$ & $41.8 \pm 10.9$ & $43.5 \pm 11.5$ & 0.06 \\
\hline Female gender & $37(49.3)$ & $34(45.3)$ & $71(47.3)$ & 0.74 \\
\hline TST negativity (<10 mm) & $63(84.0)$ & $67(89.3)$ & $130(86.7)$ & 0.47 \\
\hline Stage & & & & 0.74 \\
\hline I & $44(58.7)$ & $41(54.7)$ & $85(56.7)$ & \\
\hline II & $31(41.3)$ & $34(45.3)$ & $65(43.3)$ & \\
\hline Size of sampled lymph nodes on EBUS (short axis), mm & $15.7 \pm 3.4$ & $15.9 \pm 2.2$ & $15.8 \pm 3.3$ & 0.69 \\
\hline Size of largest lymph node sampled on EBUS (short axis), mm & $19.9 \pm 4.3$ & $20.7 \pm 5.6$ & $20.3 \pm 4.9$ & 0.36 \\
\hline Lymph nodes sampled, $n$ & $2.8 \pm 0.8$ & $2.7 \pm 0.7$ & $2.8 \pm 0.7$ & 0.37 \\
\hline Passes per node, $n$ & $2.1 \pm 0.5$ & $2.1 \pm 0.5$ & $2.1 \pm 0.5$ & 0.60 \\
\hline \multicolumn{5}{|l|}{ Stations sampled } \\
\hline $4 \mathrm{R}$ & 61 & 68 & 129 & 0.16 \\
\hline $4 \mathrm{~L}$ & 14 & 7 & 21 & 0.16 \\
\hline 7 & 73 & 71 & 144 & 0.68 \\
\hline $10 \mathrm{R}$ & 3 & 7 & 10 & 0.33 \\
\hline $10 \mathrm{~L}$ & 1 & 2 & 3 & 1.00 \\
\hline $11 \mathrm{R}$ & 11 & 6 & 17 & 0.30 \\
\hline $11 \mathrm{~L}$ & 49 & 43 & 92 & 0.40 \\
\hline \multicolumn{5}{|l|}{ Additional procedures performed } \\
\hline $\mathrm{EBB}$ & $70(93.3)$ & $71(94.7)$ & $141(94.0)$ & 1.00 \\
\hline TBLB & $62(82.7)$ & $67(89.3)$ & $129(86.0)$ & 0.35 \\
\hline
\end{tabular}

All values are presented as $n(\%)$ or mean \pm SD. EBUS, endobronchial ultrasound; TST, tuberculin skin test.

was the station 7 , followed by station $4 \mathrm{R}$ and $11 \mathrm{~L}$. The number of lymph nodes sampled and the number of passes per lymph node were similar in the 2 groups (Table 1 ). We performed EBB and TBLB in 141 (70 in group 1 and 71 in group 2) and 129 (62 in group 1 and 67 in group 2) subjects, respectively, and were not different between the 2 groups.

\section{Endpoints}

Sarcoidosis was diagnosed in 133 (65 in group 1 and 68 in group 2) subjects. Other diagnoses made on EBUSTBNA included tuberculosis $(n=11)$, metastatic carcinoma $(n=2)$, lymphoma $(n=1)$, and reactive lymphadenopathy $(n=3)$. Of the 133 subjects with sarcoidosis, granulomas were demonstrated by EBUS-TBNA in 109 $(82.0 \%)$. There was no difference $(p=0.65)$ in the diagnostic yield of EBUS-TBNA (Table 2) between the 2 groups (group 1 [52/65, 80.0\%] vs. group 2 [57/68, $83.8 \%]$ ). Of the 109 subjects diagnosed on EBUS-TBNA, EBB was performed in 103 and was diagnostic in 30 (29.1\%), while TBLB was performed in 97 subjects and was diagnostic in 49 (50.5\%). Of the remaining 24 subjects (with a non-diagnostic EBUS-TBNA), 14 had non- necrotic granulomas demonstrated in EBB or TBLB (2 on $\mathrm{EBB}, 6$ on TBLB, and 6 on both), and the remaining 10 were diagnosed on the basis of the clinicoradiological presentation and follow-up. Overall, EBB was diagnostic in $38(29.9 \%)$ of the 127 subjects with sarcoidosis in whom it was performed. TBLB, when performed, was diagnostic in $61(52.1 \%)$ of the 117 subjects with sarcoidosis.

An adequate aspirate was obtained in 129 of the 133 (97.0\%) subjects with sarcoidosis and was not different between the 2 groups (Table 2). The mean (SD) duration of the EBUS-TBNA procedure was 16.9 (4.0) $\mathrm{min}$ and was also not significantly different between the groups (16.4 [3.5] and 17.4 [4.4] $\mathrm{min}$ in groups 1 and 2, respectively; $p=0.17)$. The specimen was grossly contaminated with blood in 7 (5.3\%) subjects and was not significantly different between the 2 groups. Five complications occurred (bleeding at the puncture site in 1, pneumothorax in 2 , and hypoxemia in 2 subjects) and were not different between the 2 groups. Pneumothorax was minimal in 1 subject, while the other subject required a single-time aspiration. Pneumothorax was attributed to TBLB in both cases. 
Table 2. Study outcomes in subjects with a final diagnosis of sarcoidosis

\begin{tabular}{|c|c|c|c|c|}
\hline Diagnostic yield & $52(80.0)$ & $57(83.8)$ & $3.8(-9.3$ to 17.0$)$ & 0.65 \\
\hline \multicolumn{5}{|l|}{ Secondary outcome } \\
\hline Adequacy of the specimen & $63(96.9)$ & $66(97.1)$ & $0.14(-7.4$ to 7.9$)$ & 1.00 \\
\hline Duration of the EBUS procedure, min & $16.4 \pm 3.5$ & $17.4 \pm 4.4$ & $1.0(-0.4$ to 2.4$)$ & 0.17 \\
\hline Complications & $2(3.1)$ & $3(4.4)$ & $1.3(-6.7$ to 9.4$)$ & 1.00 \\
\hline Moderate-to-severe bleeding & 0 & 1 & & \\
\hline Hypoxemia & 1 & 1 & & \\
\hline Pneumothorax & 1 & 1 & & \\
\hline
\end{tabular}

All values are presented as $n(\%)$ or mean \pm SD unless otherwise specified. CI, confidence interval.

Table 3. Studies describing technical aspects of the EBUS-guided TBNA procedure in subjects with sarcoidosis

\begin{tabular}{|c|c|c|c|c|}
\hline Sun [11], 2015 & Prospective, observational & 120 & Number of lymph nodes and passes & $\begin{array}{l}\text { Yield increased up to } 3 \text { lymph nodes } \\
\text { sampled and up to } 5 \text { needle passes }\end{array}$ \\
\hline Muthu [8], 2016 & Randomized & 150 & $21 \mathrm{G}$ vs. $22 \mathrm{G}$ EBUS-TBNA needle & $\begin{array}{l}\text { No difference in the yield with } \\
\text { either needle }\end{array}$ \\
\hline Madan [9], 2017 & Randomized & 80 & ROSE vs. no ROSE & $\begin{array}{l}\text { No difference in the yield between } \\
\text { the } 2 \text { groups }\end{array}$ \\
\hline Oki [13], 2018 & Prospective, observational & 109 & Number of lymph nodes and passes & $\begin{array}{l}\text { Cumulative yield increased for up to } \\
4 \text { passes; not different whether } 1 \text { or } \\
2 \text { lymph nodes were sampled }\end{array}$ \\
\hline
\end{tabular}

cTBNA, conventional TBNA; EBUS, endobronchial ultrasound; ROSE, rapid on-site evaluation.

\section{Discussion}

The results of this study suggest that there is no difference in the diagnostic yield or the adequacy of the specimen obtained using EBUS-TBNA with either 10 or 20 revolutions of the needle within the node in subjects with sarcoidosis. In the current study, we enrolled subjects with suspected sarcoidosis to ensure the uniformity of the study population. Further, the yield of EBUS-TBNA in sarcoidosis is intermediate (between other diseases with enlarged mediastinal lymph nodes such as lung cancer and lymphoma); thus, there was a higher chance of a technical aspect of the procedure affecting the diagnostic yield [25].

We had hypothesized that a higher number of revolutions would increase the likelihood of the lymph node material to get aspirated into the needle lumen. However, the final yield did not differ significantly between the 2 groups. Certain points deserve special mention. First, we did not look at the yield for every pass made in either group. An average of 3 lymph nodes were sample per subject and an average of 2 passes were made per lymph node, totaling to an average of 6 passes per subject. With 6 passes it is possible that the cumulative yield tended to 
plateau equally in both groups. Thus, the effect of the number of revolutions on the yield during a single pass might have canceled out over 6 passes. Second, the time spent by the needle inside the lymph node while making a pass, mentioned as typically $<6 \mathrm{~s}$ in a previous article, was not recorded [26]. It is difficult to predict what role the time the needle resides within the lymph node played in affecting the yield in either group. Third, in our experience, we had observed that with an increasing number of revolutions made inside the node, there is a higher chance of inadvertently hitting an intranodal vessel, resulting in gross blood contamination of the aspirate. Due to this blood contamination, there is a chance of a reduced yield, as granulomas may get entangled in a blood clot. Blood clot histologic analysis, as described in a previous study, is not performed routinely at our center [27]. However, gross blood contamination occurred equally in both groups, thus falsifying our general observation.

The duration of the EBUS procedure was not different between the 2 groups. With a smaller number of revolutions performed inside the node, the 10-revolutions group would potentially have allowed shorter procedures; however, this did not occur. It is because the movement of the needle inside the node forms just a small fraction of the overall EBUS-TBNA procedure. Further, we used a single EBUS-TBNA needle for each subject. Thus, time was spent with every pass in pushing the sample out of the needle for processing onto glass slides and saline solution before the needle was made ready for the next pass. Another important point that needs discussion is that ROSE was also not available. It is possible that the use of ROSE could have helped us reduce the number of passes and the duration of the procedure. Further, ROSE can help avoid additional procedures such as EBB and TBLB if granulomas with significant necrosis can be identified, as this finding would suggest a diagnosis other than sarcoidosis, such as tuberculosis, in our setting. However, in case of finding nonnecrotic granulomas, it may still not be possible to avoid these procedures. In a tuberculosis-endemic setting, the results of EBB and TBLB can further help in differentiating sarcoidosis from tuberculosis. If nonnecrotic granulomas are identified in TBLB in cases of a normal lung (on high-resolution CT), the diagnosis is likely to be sarcoidosis [28]. On the other hand, sometimes, necrotic granulomas can be identified in TBLB (due to the larger piece of tissue obtained with lung biopsies compared to a lymph node aspirate), which helps make a diagnosis of tuberculosis.

A few studies have analyzed various technical aspects of the EBUS-TBNA procedure in subjects with sarcoid- osis (Table 3$)[8,9,11-13]$. Two prospective observational studies have shown that the diagnostic yield increases for up to 4 or 5 total passes made $[11,13]$. In the first study, the yield was also found to increase with the number of lymph nodes sampled and plateaued at 3 lymph nodes [11]. In the other study, there was no difference in the yield with sampling of either 1 or 2 lymph nodes [13]. Other studies in subjects with sarcoidosis have shown no difference in the yield with either the $21 \mathrm{G}$ or $22 \mathrm{G}$ needle or with the use of ROSE $[8,9]$. A small study of 11 subjects showed that the $19 \mathrm{G}$ conventional TBNA needle, when used with the EBUS scope, yielded tissue with better preservation of cellular architecture than that obtained with the $22 \mathrm{G}$ needle [12]. In studies performed in general populations of subjects with mediastinal lymphadenopathy (not specifically sarcoidosis) undergoing EBUS-TBNA, it has been demonstrated that the use of either suction or the stylet provided with the EBUS-TBNA needle does not affect the diagnostic yield $[16,29]$. The use of a novel flexible $19 \mathrm{G}$ needle has also been investigated in preliminary studies [30,31]. Before the present study, there was no report on the effect of the number of revolutions performed inside the lymph node on the diagnostic yield. The recent American College of Chest Physicians guidelines on the technical aspects of EBUS-TBNA mention that 5-15 agitations of the needle are typically performed while sampling a lymph node [15].

Finally, our study has certain limitations. It is a singlecenter study with a small sample size. We did not assess the yield for every pass in the 2 groups. ROSE and histological analysis of cell block specimens were not performed. However, this is the first study addressing this procedural aspect of EBUS-TBNA in any patient population. As the study included only subjects with sarcoidosis, the results may not be generalizable to patients with lung cancer or other causes of mediastinal lymph node enlargement.

In conclusion, there is no difference in the yield of EBUS-TBNA performed with either 10 or 20 revolutions of the needle inside the lymph node in subjects with sarcoidosis.

\section{Disclosure Statement}

All authors declare no conflicts of interest and financial disclosures. 


\section{Author Contributions}

S.D.: involved in patient management, statistical analysis, drafting and revising the manuscript. I.S.S., N.G., A.B., K.T.P, and
A.N.A.: involved in patient management, revising the manuscript. B.R.: involved in patient management, data collection, and revising the manuscript. R.A.: conceived the idea, involved in patient management, revising the manuscript.

\section{References}

1 Baughman RP, Culver DA, Judson MA: A concise review of pulmonary sarcoidosis. Am J Respir Crit Care Med 2011;183:573581.

2 Tremblay A, Stather DR, Maceachern P, Khalil M, Field SK: A randomized controlled trial of standard vs endobronchial ultrasonography-guided transbronchial needle aspiration in patients with suspected sarcoidosis. Chest 2009;136:340-346.

3 Navani N, Lawrence DR, Kolvekar S, Hayward M, McAsey D, Kocjan G, Falzon M, Capitanio A, Shaw P, Morris S, Omar RZ, Janes SM: Endobronchial ultrasound-guided transbronchial needle aspiration prevents mediastinoscopies in the diagnosis of isolated mediastinal lymphadenopathy: a prospective trial. Am J Respir Crit Care Med 2012;186:255-260.

4 von Bartheld MB, Dekkers OM, Szlubowski A, Eberhardt R, Herth FJ, in't Veen JC, de Jong YP, van der Heijden EH, Tournoy KG, Claussen M, van den Blink B, Shah PL, Zoumot Z, Clementsen P, Porsbjerg C, Mauad T, Bernardi FD, van Zwet EW, Rabe KF, Annema JT: Endosonography vs conventional bronchoscopy for the diagnosis of sarcoidosis: the GRANULOMA randomized clinical trial. JAMA 2013;309:2457-2464.

5 von Bartheld MB, van Breda A, Annema JT: Complication rate of endosonography (endobronchial and endoscopic ultrasound): a systematic review. Respiration 2014;87:343351.

6 Agarwal R, Srinivasan A, Aggarwal AN, Gupta D: Efficacy and safety of convex probe EBUS-TBNA in sarcoidosis: a systematic review and meta-analysis. Respir Med 2012; 106:883-892.

7 Gupta D, Dadhwal DS, Agarwal R, Gupta N, Bal A, Aggarwal AN: Endobronchial ultrasound-guided transbronchial needle aspiration vs conventional transbronchial needle aspiration in the diagnosis of sarcoidosis. Chest 2014;146:547-556.

8 Muthu V, Gupta N, Dhooria S, Sehgal IS, Bal A, Aggarwal AN, Behera D, Agarwal R: A prospective, randomized, double-blind trial comparing the diagnostic yield of 21 - and 22 -gauge aspiration needles for performing endobronchial ultrasound-guided transbronchial needle aspiration in sarcoidosis. Chest 2016;149:1111-1113.

9 Madan K, Dhungana A, Mohan A, Hadda V, Jain D, Arava S, Pandey RM, Khilnani GC, Guleria R: Conventional transbronchial nee- dle aspiration versus endobronchial ultrasound-guided transbronchial needle aspiration, with or without rapid on-site evaluation, for the diagnosis of sarcoidosis: a randomized controlled trial. J Bronchology Interv Pulmonol 2017;24:48-58.

$10 \mathrm{Hu}$ LX, Chen RX, Huang H, Shao C, Wang P, Liu YZ, Xu ZJ: Endobronchial ultrasoundguided transbronchial needle aspiration versus standard bronchoscopic modalities for diagnosis of sarcoidosis: a meta-analysis. Chin Med J (Engl) 2016;129:1607-1615.

11 Sun J, Yang H, Teng J, Zhang J, Zhao H, Garfield DH, Han B: Determining factors in diagnosing pulmonary sarcoidosis by endobronchial ultrasound-guided transbronchial needle aspiration. Ann Thorac Surg 2015;99: 441-445.

12 Biswas A, Wynne JP, Patel D, Weber M, Thakur S, Sriram PS: Comparison of the yield of 19-G eXcelon core needle to a $21-\mathrm{G}$ EBUS needle during endobronchial ultrasound guided transbronchial needle aspiration of mediastinal lymph nodes for the detection of granulomas in cases of suspected sarcoidosis. J Thorac Dis 2017;9:E864-E866.

13 Oki M, Saka H, Ando M, Nakashima H, Shiraki A, Murakami Y, Kogure Y, Kitagawa C, Kato T: How many passes are needed for endobronchial ultrasound-guided transbronchial needle aspiration for sarcoidosis? A prospective multicenter study. Respiration 2018;95:251-257.

14 van der Heijden EH, Casal RF, Trisolini R, Steinfort DP, Hwangbo B, Nakajima T, Guldhammer-Skov B, Rossi G, Ferretti M, Herth FF, Yung R, Krasnik M: Guideline for the acquisition and preparation of conventional and endobronchial ultrasound-guided transbronchial needle aspiration specimens for the diagnosis and molecular testing of patients with known or suspected lung cancer. Respiration 2014;88:500-517.

15 Wahidi MM, Herth F, Yasufuku K, Shepherd RW, Yarmus L, Chawla M, Lamb C, Casey KR, Patel S, Silvestri GA, Feller-Kopman DJ: Technical aspects of endobronchial ultrasound-guided transbronchial needle aspiration: CHEST guideline and expert panel report. Chest 2016;149:816-835.

16 Casal RF, Staerkel GA, Ost D, Almeida FA, Uzbeck MH, Eapen GA, Jimenez CA, Nogueras-Gonzalez GM, Sarkiss M, Morice RC: Randomized clinical trial of endobronchial ultrasound needle biopsy with and without aspiration. Chest 2012;142:568-573.
17 Kaur H, Dhooria S, Aggarwal AN, Gupta D, Behera D, Agarwal R: A Randomized trial of 1 versus $2 \%$ lignocaine by the spray-as-yougo technique for topical anesthesia during flexible bronchoscopy. Chest 2015;148:739745 .

18 Dhooria S, Sehgal IS, Gupta N, Aggarwal AN, Behera D, Agarwal R: Diagnostic yield and complications of EBUS-TBNA performed under bronchoscopist-directed conscious sedation: single center experience of 1004 subjects. J Bronchology Interv Pulmonol 2017;24:7-14.

19 Dhooria S, Agarwal R, Aggarwal AN, Bal A, Gupta N, Gupta D: Differentiating tuberculosis from sarcoidosis by sonographic characteristics of lymph nodes on endobronchial ultrasonography: a study of 165 patients. J Thorac Cardiovasc Surg 2014; 148:662-667.

20 Dhooria S, Agarwal R, Aggarwal AN, Gupta N, Gupta D, Behera D: Agreement of mediastinal lymph node size between computed tomography and endobronchial ultrasonography: a study of 617 patients. Ann Thorac Surg 2015;99:1894-1898.

21 Sehgal IS, Bal A, Dhooria S, Agrawal P, Gupta N, Ram B, Aggarwal AN, Behera D, Agarwal R: A prospective randomized controlled trial comparing the efficacy and safety of cup vs alligator forceps for performing transbronchial lung biopsy in patients with sarcoidosis. Chest 2016;149:1584-1586.

22 Dhooria S, Gupta N, Bal A, Sehgal IS, Aggarwal AN, Sethi S, Behera D, Agarwal R: Role of Xpert MTB/RIF in differentiating tuberculosis from sarcoidosis in patients with mediastinal lymphadenopathy undergoing EBUS-TBNA: a study of 147 patients. Sarcoidosis Vasc Diffuse Lung Dis 2016;33:258266.

23 Costabel U, Hunninghake GW: ATS/ERS/ WASOG statement on sarcoidosis. Sarcoidosis Statement Committee. American Thoracic Society. European Respiratory Society. World Association for Sarcoidosis and Other Granulomatous Disorders. Eur Respir J 1999;14:735-737.

24 Statement on sarcoidosis: Joint statement of the American Thoracic Society (ATS), the European Respiratory Society (ERS) and the World Association of Sarcoidosis and Other Granulomatous Disorders (WASOG) adopted by the ATS Board of Directors and by the ERS Executive Committee, February 1999. Am J Respir Crit Care Med 1999;160:736755 . 
25 Dhooria S, Sehgal IS, Aggarwal AN, Agarwal R: Convex-probe endobronchial ultrasound: a decade of progress. Indian J Chest Dis Allied Sci 2016;58:21-35.

26 Murgu SD: Diagnosing and staging lung cancer involving the mediastinum. Chest 2015;147:1401-1412.

27 Amin EN, Russell CD, Shilo K, Islam S, Wood KL: Diagnostic value of blood clot core during endobronchial ultrasound-guided transbronchial needle aspirate. Lung 2013; 191:305-309.
28 Gupta D, Agarwal R, Aggarwal AN, Jindal SK: Sarcoidosis and tuberculosis: the same disease with different manifestations or similar manifestations of different disorders. Curr Opin Pulm Med 2012;18:506-516.

29 Scholten EL, Semaan R, Illei P, Mallow C, Arias S, Feller-Kopman D, Oakjones-Burgess K, Frimpong B, Ortiz R, Lee H, Yarmus L: Stylet use does not improve diagnostic outcomes in endobronchial ultrasonographic transbronchial needle aspiration: a randomized clinical trial. Chest 2017;151:636-642.
30 Tyan C, Patel P, Czarnecka K, Gompelmann D, Eberhardt R, Fortin M, MacEachern P, Hergott CA, Dumoulin E, Tremblay A, Kemp SV, Shah PL, Herth FJF, Yasufuku K: Flexible 19-gauge endobronchial ultrasound-guided transbronchial needle aspiration needle: first experience. Respiration 2017;94:52-57.

31 Czarnecka-Kujawa K, Tremblay A, Yasufuku K, Sczaniecka A, Parthiban S, Kunz L, Dillard D, Gonzalez X: A preclinical evaluation comparing the performance of a novel 19-G flexible needle to a commercially available 22-G EBUS-TBNA sampling needle. Respiration 2018;95:55-62. 